\title{
An Integrated And Cohesive Methodology For Smart Manufacturing Application Based On Cloud, Edge And Fog Computing
}

\author{
R. Veeramani ${ }^{1}$, Dr.R.MadhanMohan ${ }^{2}$, Dr.C.Mahesh ${ }^{3}$ \\ ${ }^{1}$ Assistant Professor, Department of Information Technology, SRM Institute of Science and Technology, Ramapuram, Chennai, \\ India. gvmani.r@gmail.com \\ ${ }^{2}$ Associate Professor, Department of Computer Science and Engineering, Annamalai University, Annamalai Nagar, \\ Chidambaran, India. madhanmohan_mithu@yahoo.com \\ ${ }^{3}$ Associate Professor \& Head, Department of Information Technology, Vel Tech Rangarajan Dr. Sagunthala R\&D Institute of \\ Science and Technology, Chennai, India, chimahesh@gmail.com
}

\begin{abstract}
Cloud fabricating is rising as a key empowering influence for assembling organizations to convey exceptionally customizable administrations over the Internet. This paper means to examine how cloud fabricating frameworks can encourage viable assistance situated business [8]. This framework proposes a cloud-based pluggable assembling administration plot by utilizing distributed computing, RESTful Web Service and edge registering to tackle this issue [7]. By making use of a double layer administration instrument, the assembling administrations can be worked as pluggable application module [8]. The suggested System enables the designers to convey chose Pluggable Application Modulations from the cloud to the chosen edge gadgets productively and to run and deal with the stopped PAMs remotely through the cloud stage utilizing Web-based GUIs for supporting keen assembling exercises on the chosen creation gear [11]. In the suggested framework, the key viewpoints are improved assembling system perceivability, data sharing, fabricating process coordination and inconstancy, and educated basic leadership with the goal that the general creation runs as per plan [13].
\end{abstract}

Key words: PAM, Cloud, GUI, Framework, Perceivability, Data sharing, Inconstancy, Services, Leadership.

\section{INTRODUCTION}

The Internet of Things (IoT) is one amongst the astronomical trends in technology. About twenty six billion tools were installed by 2020 in a rapid speed. Data to be handled and scrutinized are effectuated in substantial consignment. The proceedings and the investigation of the IOT data systematically increase proportional to the capacity of the conservatory. This leads to diagnostic provocation and stipulates large number of computing equity.

In an attempt to give a discourse on the requisitions and meet the insistence of enumerating benevolence, an umpteen of all-encompassing data centers or clouds have been manipulated. For good measure, cloud computing has been propounded to endow fiscal and productive indulgence to the clients. Here, for the benefit of the user's ingress, the cloud computing enables the cloud data centers that allow partnered stakes of customizable computing sources. This results in ascending dissemination expenditure and congestion since the clouds are erected in outback areas. For applications requiring coetaneous significance, it is insupportable.

Engrossing the clouds to the users proves to be more advantageous. The representation of fog computing is to impel data to the data centers. It allows computing devices with low-power to perform action, decision-making and computing. Such components are named as fog nodes. Hence fog computing provides location cognizance and rapid- response.

For presuming astronomical applications to the users, an enormous number of data centers at different locations are possessed by the fog computing networks. Fog computing effectuates the network emulation that leads to indiscernibility of fog nodes to the clients. For refinement of extravagant capacity, the clients can attain only the resources of computing from data centers of the cloud. Agglomeration of computing resources from fog nodes are been able to do by each cloud data centers upon acquiring all the aspirations of the clients. This allows the clients to adopt the resources of computing in a coherent manner. The main hindrance to this is the dispensation of restricted resources of computing from all fog nodes to the clients. As a consequence of this, our paper is appositely designed for potential technique of smart manufacturing to ascend the efficiency of cost.

\section{RELATED WORKS}

Now-a-days, with the increased development of technology has led for it to be greatly applied into the industrial fields such as electricity, transportation, petroleum, metallurgy, military, chemical, manufacturing and so on [1]. By gradually introducing various sections such as Internet, WSN, embedded system, Internet, smart terminal and software for control and management makes 
the previously existing industrial system more aware, supporting to the upcoming revolution of the industry [4]. This paper discusses mainly about the issues of the cloud Operating System for application in the industry, including the cloud computing along with cloud operating system, resulting in its analysis of cloud OS and its transformation [7]. The application and its result are obtained by independently designing the content of the cloud [13]. This study provides challenges and guidance for the creation of cloud OS oriented to industrial area [6].

System intelligence is obtained from the data acquired. By making use of the integrated analysis all aspects of manufacturing [9] are boosted resulting in increased production. Smart manufacturing [1] can be achieved by paving a way for the cyber-physical combination of the manufacturing process [5]. The concept of big data and digital double and their applications in product style, production scheming, creation [1], and predictive support are reviewed. To promote the smart manufacturing process [4], the main differences as well as the similarities are analyzed and compared to better complement so that they can be integrated into the system.

Smart coordination is one of the most crucial concerns for implementation as smart creation process has become the common goal for various countries [9]. Smart coordination cannot be realized by dealing with current devices, layout and its deployment. In order to resolve these issues, the IIHub consisting of the CA-Module [9]. The physical manufacturing resources can be connected by configuring flexible CA-Modules [5]. The deployment for smart interconnection and quick configuration can be supported by making use of the services provided by the IIHub [9]. An account is then provided to better exemplify the various tasks of the suggested IIHub, as well as to display how smart interconnection can be realized using IIHub.

Even though it has not attracted attention in the vehicular sector [10], the cloud radio access network is regarded as the most promising field for the next generation of mobile networks [8]. The inherent "clouddown" design is most commonly used as a computational capability for universal radio resource optimization in mobile networks [7]. On the other hand the "edge-up" design is preferred so as to not only rely on wireless infrastructure to promise safety. The most vital function of this article is to assist all possible wireless applications for the next age of driving machines. The suggested designs enable us to develop a field shift so as to aid in the creation of software-defined mobile-edge vehicular networking [10].

Now-a-days, many countries issued their development of various strategies, such as Industrial Internet, Industry 4.0, and created in China 2025 along with the wide implementation of New IT in manufacturing [5]. The most common goal of using these strategies is to achieve smart manufacturing [1]. New IT has played crucial roles to promote smart manufacturing [9]. To adjust the physical world the data can be analyzed and processed by cloud computing [6] by transferring data from physical to the cyber world and vice-versa through the Internet \& IOT [3]. The latest trend in manufacturing process is servitization [5]. The most widely used service for users is the concept of "Manufacturing-as-a-Service"[4]. Due to platform independence and interoperability, services lead the way for collaboration and creation of smart applications. This paper proposes service-oriented smart manufacturing (SoSM) [4] which is a combination of New IT and services. Its main goal is to facilitate smart manufacturing [9] using services along with New IT. In addition to the typical characteristics of SoSM, the SoSM framework is also discussed and investigated [12].

\section{EXISTING SYSTEM}

The current framework comprises of all the assembling assets associated with different assembling exercises [12]. In a shop-floor or production line, head assets can be outlined as "Human-Machine-MaterialEnvironment" [1]. Human alludes to laborers and chiefs. Contraption incorporates machining gear (e.g., contraption instruments, robots, and so forth.), shipping hardware (e.g., the material transports, programmed automobiles, and so on.) [10]. Material comprises of crude material, parts and segments, semi-completed items, completed items, and so on [2].

In the current framework, is liable for medium speed and idleness information examination for assembling data joining and the board [12]. The parts (e.g., arrange switches, local servers, organize switches, and so forth.) of edge or center system, and venture class the board frameworks could be utilized as the foundations for mist registering [2]. The mist the hub of executives can know about all the mist hubs in a mist arrange. The haze hub speaks with the cloud server farm of the outside system through the haze the board hub [7]. In mist layer, edge association gadgets and edge figuring gadgets speaks with the haze door through a solitary jump low-inactivity connect. The inward haze hubs speak with the edge layer through the mist portal. The primary capacity of the internal mist hub is to perform information handling (information separating, pressure, stockpiling, encryption, and so forth.) on the information from the edge layer and preparing administration demands.

Information created by different assembling assets, which might be topographically appropriated, is encountering unstable development. This information are passed on over the system to cloud, where information handling is done. The expanding volume and speed of information require high transfer speed, which is over the top expensive. At the point when the system clog is not kidding, little information might be misfortune.

\section{DRAWBACKS OF EXISTING SYSTEM}

\footnotetext{
$>$ Maintenance cost of expensive computing infrastructure.

$>$ The file processing requires high reasonableness.

$>$ High costs in the cellular networks.

$>$ Lacks all kinds of complex product design.
} 


\section{PROPOSED SYSTEM}

The suggested framework manages multi-source, multi-scale and multivariate information [12]. Multisource information: various data sources are engaged with information assortment, going from Internet-of-Things (IoT) and control frameworks [12], to Cyber-Physical Systems (CPS), Enterprise Information Systems (EIS) and many other assembling resources (e.g.,contraption, creation sentences, storage, and so on).

Multi-scale information: Different kinds of information from different activities and procedures are included, which length over numerous degrees of the mechanical mechanization progression, from low level machines, to generation lines and store network systems, up to the entire processing plant computerized world. Multivariate information: The information show various factors, through which it tends to be considered and handled, for example, fleeting perspectives that influence the choice of the suitable assortment components or the objective assets that this information is alluded as for the choices that should be made.

In the proposed framework, the utilization case driven adaptable total of keen assembling information, brilliant assembling involves progressively exact and far reaching control on circumstance mindfulness for the leaders and the partners in the business to empower them evaluate the effect of interruptions on the present generation and store network abilities.

\section{ADVANTAGES OF PROPOSED SYSTEM}

$>$ The data is generated and renewed at very high speed.

$>$ Improving the planning speed and accuracy.

$>$ Highly efficient communication contracts in the form of XML.

$>$ Low cost along with high efficiency.

$>$ Cloud computing mechanism is more available and reliable [13].

\section{SYSTEM ARCHITECTURE}

There are three layers in this fog computing network which includes fog layer, client layer and cloud layer. Extensive cloud data centers with huge count of performance in computing units are occupied in the cloud layer. Engrossing the users of the fog nodes are collectively occupied in the fog layer. Low- power devices of computing occupy the client layer.

The fog nodes are positioned in closer precincts of the clients. This allows clients to succumb their capacity to the engrossed fog nodes. This capacity succumbed by the clients are to be designated efficiently since there are minimum power resource and computing. Through parochial communication structure, the fog nodes can interact with each other in a cooperative manner. This allows the workload processing in a combined manner by helping one other, so that the quality of service is improvised. These layers include the functioning of cloud, edge and fog computing.

Now that the layers of these are constructed, every client provides the services of computing to the nearby fog nodes which are assembled by the data centers of the cloud. Let us contemplate the number of data centers of the cloud to be " $\mathrm{D}$ ". These can be expressed as follows:

$$
\mathrm{N}=\{\mathrm{n} 1, \mathrm{n} 2, \mathrm{n} 3, \mathrm{n} 4, \ldots \ldots \ldots \ldots \ldots \mathrm{nD}\}
$$

The unacknowledged network will contain "M" data centers which can also be expressed as follows:

$$
\mathrm{A}=\{\mathrm{a} 1, \mathrm{a} 2, \mathrm{a} 3, \mathrm{a} 4, \ldots \ldots \ldots \ldots \ldots . . \mathrm{aM}\}
$$

Every cloud data centers has the rights to withdraw the services given by the clients to reduce the adjourn and agile antiphon of resources. Let us deem that the number of fog nodes in the network to be "L" which is labeled as:

$$
\mathrm{C}=\{\mathrm{c} 1, \mathrm{c} 2, \mathrm{c} 3, \mathrm{c} 4, \ldots \ldots \ldots \ldots \ldots . \mathrm{cL}\}
$$

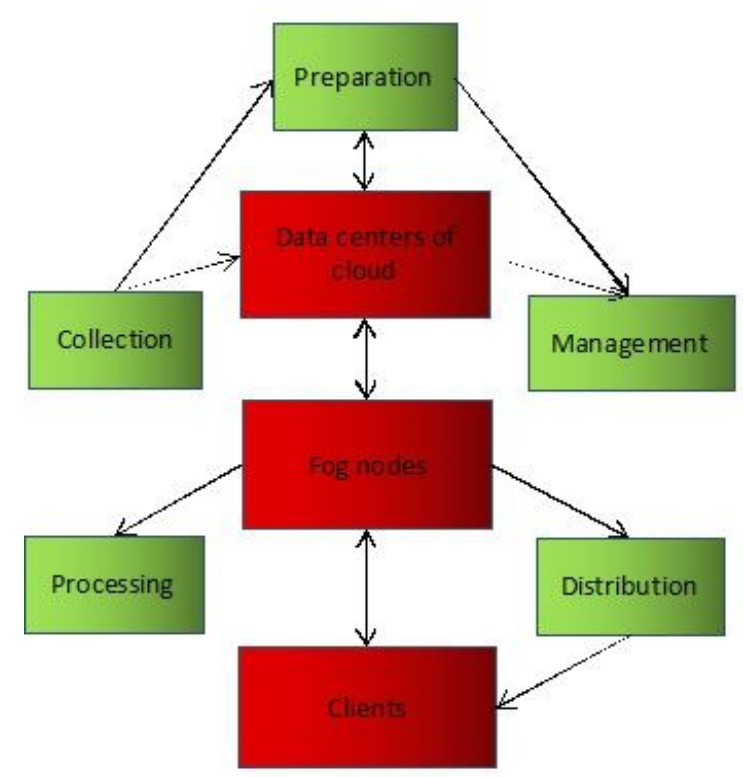

Figure-1: System architecture

The interdependence between the fog nodes, clients and the data centers are done by the processes described in the given figure. This process originates from the data collection from the fog nodes. The allied reciprocity of data are oppressed unmitigatedly. Therefore the capabilities of the computation of the edge devices are monopolized triumphantly. In the meanwhile, the performance of reconstructed algorithm with astronomical probability turn out to be pecunious which are in turn assured by the schemes of encoding and decoding among the fog, cloud and edge computing.

Once the data from the fog nodes are being collected, they are set for processing of the data. There are three basic processing. They are course, aggregation and serveless processing. This processing involves huge accomplishments which will not be a part of the edge 
R. Veeramani et al., International Journal of Advanced Trends in Computer Science and Engineering, 10(1), January - February 2021,289 - 294

since, it has to work on enormous data and to make it cost efficient.

\subsection{Integrated Methodology In Cloud, Edge And Fog Computing}

The predilection rendering and the evaluation of collateral disbursement issue strategy uses the norms that is desired in account of the reciprocity of collateral disbursement issue and fog computing [5]. Only the collateral disbursement issue in fog computing either ascending the quality of service or descending the amount comprises the existing system. There are two censorious norms which are been considered concurrently in this paper. The levy coherence norm of the network is defined by the proportion of convenience to the total cost apart from the hypothesis of levy coherence that reduces the total cost parallel to the satisfaction of quality of service which is given as:

\section{Pce $=$ Convenience of service quality / Levy of service quality.}

The network circuit-clout capacity emanation time and the echelon delay at the fog layer include the quality of service that is calculated by the delay service. The capacity of each client is taken into account using the Poisson arrival process. The deviation of the returns acquired from the clients and the remittance to the premises serves as the profitability of each cloud data center [4]. On the essence of quantity of constructive data centers, they are foisted to the fog nodes on combining the data centers and the fog nodes. Even if the fog node does not produce the required computing resource block, the clients will be succoured by the data centers [14].

The procurement of quintessential quantity of constructive resource block from data centers by the clients is made as an assumption. The coalition of data center and fog nodes and that of the coalition of the fog node and the client are to be integrated on the basis of cost efficiency [5]. Therefore, on the essence of mesh norm maximization, the dual coalition problems are sanctioned as a binary-integer programming problem. By continuous recurrence of the above process, the final result would be obtained.

\section{MODULES}

\subsection{Module 1: Amazon Data Extraction}

Python application, script or library with AWS services are easily integrated using Boto3 [4]. The object oriented interface in the Amazon web services are represented using the resources provided by Boto3 [6]. They supply a greater-level abstract than the low-level calls provided by the clients of the service [11]. The method and attributes are present in each and every resource. The attributes, identifiers, references, actions, collections and sub-resources are separated conceptually. The AWS APIs are called by the Boto3 on our behalf. Two different ways offered by Boto3 for accessing these APIs are: Client: low-level service access [6] Resource: higher-level object-oriented service access. These resources can be split into service resources and individual resources. Service resources don't contain attributes or identifiers [11]. Actions can be used on the resource by using an identifier. At least one identifier must be present in a resource. If the production of identifiers which is about at instance creation-time fails during instantiation, it would result in the creation of an exception. An action could be used to make a call to the service [4]. Actions may return a low-level response either a listing of recent resource instances or a brand new resource instance. Even though resource identifiers are automatically set as parameters by the actions, the supplementary parameters could be passed via argument. There are no common identifiers between the reference resource and the resource instance, i.e., it's not a demanding parent to child relationship. In relational terms, these will be considered many-to-one or one-to-one.

\subsection{Module 2: Azure Data Extraction}

The Azure SDK for Python simplifies using and managing Azure resources from Python application code. A number of client libraries in the SDK help you connect to existing Azure resources and use them in your apps, such as uploading files, accessing table data, or working with the various Azure Cognitive Services [6]. With the SDK you work with those resources using familiar Python programming paradigms rather than using the service's generic REST API [4].

The Azure SDK for Python also includes many libraries to help you create, provision, and otherwise manage Azure resources themselves. We refer to these as management libraries [6]. Each management library is named azure-mgmt-<service name> [11]. With the management libraries, you can write Python code to accomplish the same tasks that you can using the Azure portal or the Azure CLI [7].

The Azure command-line interface (Azure CLI) is an environment to create and manage Azure resources. Azure CLI is available across Azure services [4] and is designed to get you working quickly with Azure, with an emphasis on automation. Automated tools that use Azure services should inevitably have limited permissions [6]. Instead of having applications sign in as a fully privileged user, Azure offers service principals [11].

In order to gain entrance to Azure resources, the Azure service principal is created to be used in conjecture with the applications, hosted services, and automated tools. The access to the Azure resources are restricted by the various tasks provided to the Azure service principal, thereby giving the user authority over which resources can be used and at which juncture [4]. For security reasons, it's always recommended to use service principals with automated tools rather than allowing them to $\log$ in with a user identity. 
R. Veeramani et al., International Journal of Advanced Trends in Computer Science and Engineering, 10(1), January - February 2021,289 - 294

\subsection{Module 3: Exploratory Data Analysis}

Even though EDA and statistical graphics are not similar, they are often used in conjecture to each other. Statistical graphics can be defined as a collaboration of techniques each based graphically and specializing in a single data aspect. Exploratory Data Analysis allows the user to understand and comprehend the fundamental prototype of the data thereby postponing the pre-existing assumptions of the information. Exploratory Data Analysis is defined as a theory on how to study data set; what to look for; how it is looked upon; and the way we comprehend. It is widely known that EDA frequently uses a combination of techniques which is known as "statistical graphics".

Statistical graphics makes use of the data that is acquired through making a specific measurement in all genres of a sample to display our findings for a single aspect such as generation, type, completion time, or reaction to a stimulus. These measurements constitute a "sample distribution" of the variable, which constitutes the "population distribution" of the variable. The standard goal of EDA is to acknowledge the "sample distribution" and also to create some definitive conclusions about which "population distribution" is compatible with the sample. Outlier detection is additionally a component of this examination.

\subsection{Module 4: Cost Forecasts}

The cost model governs the relationship between a resource allocation/reconfiguration decision and the associated penalty/cost incurred by the application host. It comprises of resource cost, reconfiguration cost due to service degradation or downtime during reconfiguration actions, and monetary Income associated with providing a particular level of service [6]. Using these components, we formulate two types of models corresponding to satisfying Service Level Agreement (SLA) and profit maximization.

Resources that provide allocation lower than the task requirements are penalized in terms of monetary cost. However, the degree to which the resource is penalized depends both on how far the allocation is from the required value (as captured using $\rho$ ), as well as an exponential penalty factor

\section{DEMO GRAPHIC}

A diegesis is contemplated to get the measure of contemporary facsimile results and production using fog computing networks [3]. Here, the mobile devices with data processing meticulous applications are claimed as clients. Access points that are managed by the access points are termed as fog nodes as far as this diegesis is considered. The graph shows the various parameter values. The value of indulgence quotient of each computing resource block is $0.2 \mathrm{~m} / \mathrm{s}$. And the average capacity arriving rate quotient is $1.0 \mathrm{~m} / \mathrm{s}$. On the basis of efficiency of cost, the predilection of each fog node is set to each data center of the cloud.

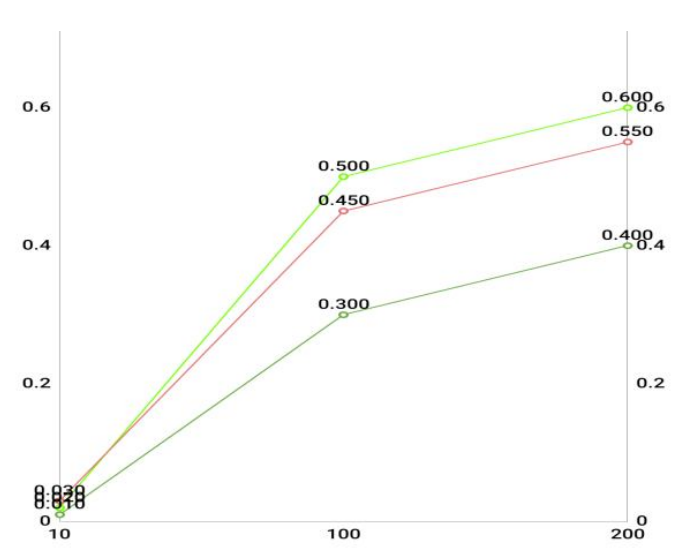

Figure-2: Efficiency of cost versus count of clients on the basis of capacity levels.

The efficient of cost with the count of clients are been represented in the Figure.2. This representation clearly proves that the counts of clients are ascending gradually. The comparison of cost efficiency pursuance with numerous capacity is been accomplished here. As the prosaic capacity for each client ascends, the cost efficiency pursuance reaches a peak value.

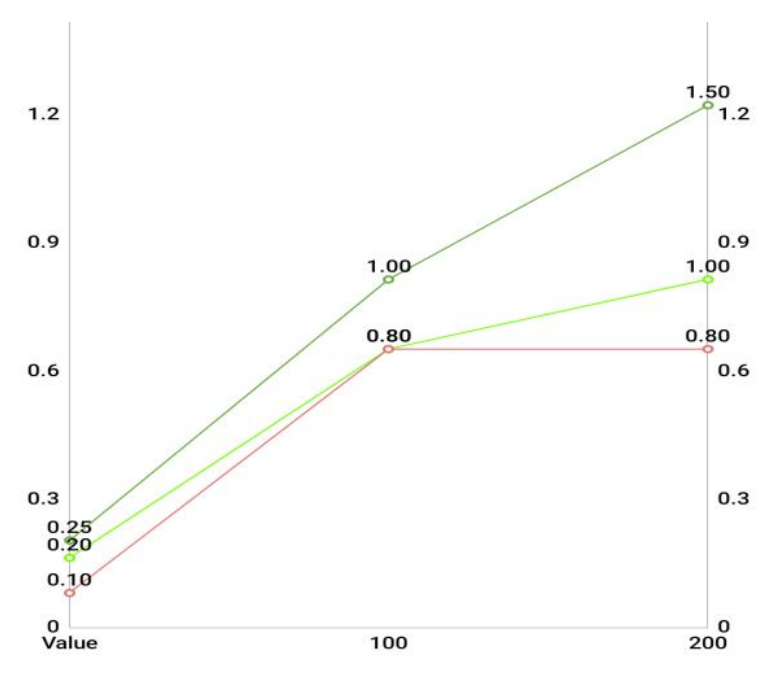

Figure-3: Efficiency of cost versus count of clients on the basis of number of fog nodes.

As the count of clients ascends according to the astronomical quantity of fog nodes, the cost efficiency pursuance is been calibrated in Figure.3. With the accentuating count of clients, the cost efficiency gradually ascends amid the fog nodes are static. The accelerating speed gradually accentuates and then descends to zero gradually. It is due to the fact that the fog nodes allocate more clients when the count of clients accentuates. When this process is accomplished, the clients would be further served from the data centers of the cloud [13]. 


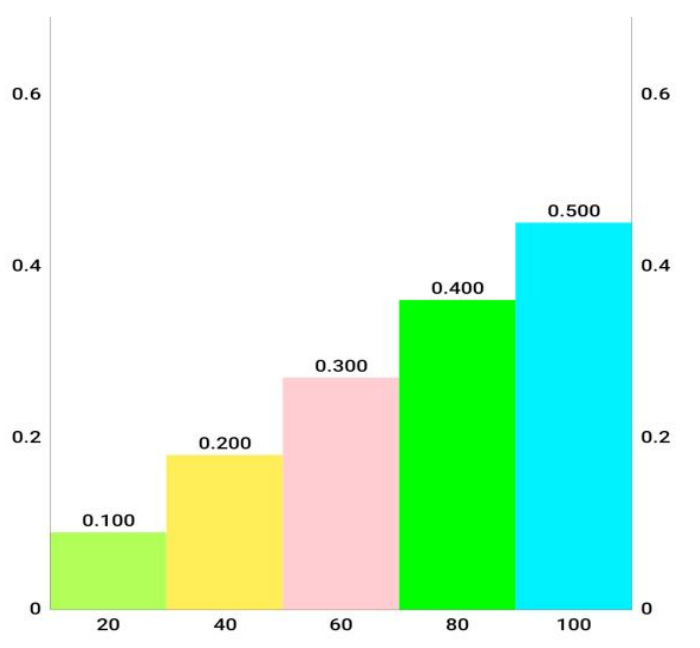

Figure-4: Bar graph analysis of client count with rate of service.

The fog nodes cease to ascend in sequence with the pursuance of cost efficiency based on Figure.4. Clients can be conglomerated at a higher rate with assistance of fog nodes when the client count becomes astronomical [5]. Now, the ascending speed becomes minimum.

\section{CONCLUSION}

For manufacturing companies to stay competitive, producing new PSS utilizing distributed resources based on a pay-as-you-go model is an effective approach. There are still a number of technical challenges in transforming today's manufacturing systems into service-oriented manufacturing models under a cloud manufacturing environment. The suggested cloud-based system can facilitate service-oriented manufacturing business in a decentralized and dynamic manufacturing environment. The importance of data-driven service orchestration in improving the decision making processes in smart manufacturing ecosystems has been highlighted.

\section{REFERENCES}

1. F. Tao and Q. Qi, “New IT driven service-oriented smart manufacturing: Framework and characteristics," IEEE Trans. Syst., Man, Cybern. Syst., vol. 49, no. 1, pp. 8191, Jan. 2019.

2. C. Zhu, V. C. M. Leung, L. Shu, and E. C.-H. Ngai, “'Green Internet of Things for smart world," IEEE Access, vol. 3, pp. 21512162, Nov. 2015.

3. L. Da Xu, W. He, and $\mathrm{S} . \mathrm{Li}$, "Internet of Things in industries: A survey," IEEE Trans. Ind. Informat., vol. 10, no. 4, pp. 22332243, Nov. 2014.

4. F. Tao, Q. Qi, A. Liu, and A. Kusiak, “Data-driven smart manufacturing," J. Manuf. Syst., vol. 48, pp. 157169, Jul. 2018.

5. J. Pizo « and J. Lipski, “Perspectives for fog computing in manufacturing," Appl. Comput. Sci., vol. 12, no. 3, pp. 3746, 2016.

6. F. Tao, L. Zhang, Y. Liu, Y. Cheng, L. Wang, and X. Xu, "Manufacturing service management in cloud manufacturing: Overviewand future research directions," J. Manuf. Sci. Eng., vol. 137, no. 4, p. 040912, 2015.
7. Y. Liu and X. Xu, “Industry 4.0 and cloud manufacturing: A comparative analysis," J. Manuf. Sci. Eng., vol. 139, no. 3, p. 034701, Mar. 2017.

8. F. da Silva, R. L. Ohta, M. N. D. Santos, and A. P. D. Binotto, "A cloud-based architecture for the Internet of Things targeting industrial devices remote monitoring and control," IFACPapersOnLine, vol. 49, no. 30, pp. 108113, 2016.

9. G. Xiong, T. Ji, X. Zhang, F. Zhu, and W. Liu, “Cloud operating system for industrial application," in Proc. IEEE Int. Conf. Service Oper. Logistics, Informat., Nov. 2016, pp. 4348.

10. F. Tao, J. Cheng, and Q. Qi, “'IIHub: An industrial Internet-of- Things hub toward smart manufacturing based on cyber-physical system," IEEE Trans. Ind. Informat., vol. 14, no. 5, pp. 22712280, May 2018.

11. D.-J. Deng, S.-Y. Lien, C.-C. Lin, S.-C. Hung, and W.-B. Chen, "Latency control in software-defined mobile-edge vehicular networking," IEEE Commun. Mag., vol. 55, no. 8, pp. 8793, Aug. 2017.

12. F. Tao, Y. Cheng, L. D. Xu, L. Zhang, and B. H. Li, "CCIoTCMfg: Cloud computing and Internet of Things-based cloud manufacturing service system," IEEE Trans. Ind. Informat., vol. 10, no. 2, pp. 14351442 , May 2014

13. H. Lee, "Framework and development of fault detection classification using IoT device and cloud environment," J. Manuf. Syst., vol. 43, pp. 257270, Apr. 2017.

14. D. Mourtzis, E. Vlachou, N. Xanthopoulos, M. Givehchi, and L. Wang, "Cloud-based adaptive process planning considering availability and capabilities of machine tools," J. Manuf. Syst., vol. 39, pp. 18, Apr. 2016.

\begin{tabular}{|l|l|}
\hline & \\
& \\
& \\
&
\end{tabular}

